\title{
Imaging of RNA in situ hybridization by atomic force microscopy
}

\author{
W. H. J. KALLE,* M. V. E. MACVILLE,*1 M. P. C. VAN DE CORPUT,* B. G. DE GROOTH, $\dagger$ \\ H. J. TANKE* \& A. K. RAAP* \\ *Department of Cytochemistry and Cytometry, Leiden University, Wassenaarseneg, 72, \\ 2333 AL Leiden, The N etherlands \\ †Department of Applied Physics, University of Twente, PO Box 217, 7500 AE Enschede, \\ The N etherlands
}

Key words. A tomic force microscopy, cell morphology, DAB, ethanol dehydration, in situ hybridization, peroxidase, RNA.

\section{Summary}

In this study we investigated the possibility of imaging internal cellular molecules after cytochemical detection with atomic force microscopy (AFM). To this end, rat $9 \mathrm{G}$ and HeLa cells were hybridized with haptenized probes for $28 \mathrm{~S}$ ribosomal RNA, human elongation factor mRNA and cytomegalovirus immediate early antigen mRNA. The haptenized hybrids were subsequently detected with a peroxidase-labelled antibody and visualized with $3,3^{\prime}$ diaminobenzidine (DAB). The influence of various scanning conditions on cell morphology and visibility of the signal was investigated. In order to determine the influence of ethanol dehydration on cellular structure and visibility of the DAB precipitate, cells were kept in phosphate-buffered saline (PBS) and scanned under fluid after DAB development or dehydrated and subsequently scanned dry or submerged in PBS. Direct information on the increase in height of cellular structures because of internally precipitated DAB and the height of mock-hybridized cells was available. Results show that internal DAB precipitate can be detected by AFM, with the highest sensitivity in the case of dry cells. Although a relatively large amount of DAB had to be precipitated inside the cell before it was visible by AFM, the resolution of AFM for imaging of RNA - in situ hybridization signals was slightly better than that of conventional optical microscopy. Furthermore, it is concluded that dehydration of the cells has irreversible effects on cellular structure. Therefore, scanning under fluid of previously dehydrated samples cannot be considered as a good representation of the situation before dehydration.

\footnotetext{
${ }^{1}$ Present address: Diagnostic Development Branch, National Centre for Human Genome Research, NIH, Bldg 49, Bethesda MD 20892, U.S.A. Correspondence: W. H. J. Kalle. Tel. 31 71276190; Fax 3171276180.
}

\section{Introduction}

Atomic force microscopy (AFM), a form of scanning probe microscopy, is a relatively new type of microscopy invented in 1986 (Binnig et al., 1986). A sharp tip, mounted on a cantilever with a low spring constant is raster scanned over a surface Forces acting between the atoms from the surface and the atoms at the end of the tip will cause deflection of the cantilever. Measurements of the displacement of the cantilever are displayed in a two-dimensional matrix, giving a topographic representation of the sample. AFM is capable of imaging the topography of biological samples in air and under fluid. In principle, living cells can be studied under biologically relevant conditions.

A limitation of AFM is that only information from the surface of the sample is detected. When the targets are too small or located inside the sample, a topographical hallmark has to be created on top of the target, after which AFM can be used to detect the hallmark (Putman et al., 1993a,b; Rasch et al., 1993). In the past few years, a large number of studies has been reported that show the use of AFM for imaging of cells, subcellular structures and macromolecules such as DNA, RNA and proteins (reviewed by Hansma \& Hoh, 1994; Yang \& Shao, 1995). The inside of cells can be imaged by removing the membrane, thus exposing cytoplasm and cytoplasmic structures (Pietrasanta et al., 1994). When relatively soft samples like cells are imaged, deformation of the cell membrane may occur, thereby revealing relatively hard features, e.g. cytoskeleton and nucleoli, under the cell membrane (Henderson et al., 1992; Keller et al., 1992; Putman et al., 1993c).

In order to investigate the possibility of visualizing internal cellular molecules with AFM, we used an in situ hybridization procedure in combination with peroxidase diaminobenzidine staining for the detection of differently expressed 
cytoplasmic and nuclear RNAs. As model systems for the detection of RNA - in situ hybridization (ISH) signals with A FM we visualized human elongation factor (HEF) MRNA on HeLa cells, human cytomegalovirus (HCMV) immediate early antigen (IE) mRNA on rat $9 \mathrm{G}$ cells and $28 \mathrm{~S}$ ribosomal RNA (rRNA) on both cell types. These RNAs have been localized previously with fluorescence microscopy (Dirks et al., 1993), reflection contrast microscopy and electron microscopy (Macville et al., 1995). Different scanning conditions to improve the visibility of the ISH signal and the structure of the cells after the ISH procedure were also tested. A balance was found between the visibility of the signal, which ben efits from large amounts of 3,3'-diaminobenzidine (DAB) precipitate and optimal spatial resolution, for which as little DAB precipitate as possible is indicated. The advantages of the potentially higher resolution of AFM than that of conventional optical microscopy in these type of studies is discussed.

\section{Materials and methods}

\section{Cell culture}

Rat $9 \mathrm{G}$ and HeLa cells were cultured to subconfluency on sterilized glass slides in Dulbecco's minimal essential medium (DMEM) supplemented with 10\% fetal calf serum, $0.03 \%$ glutamine and $10000 \mathrm{U} \mathrm{mL}^{-1}$ penicillin/streptomycin at $37{ }^{\circ} \mathrm{C}$ in a $5 \% \mathrm{CO}_{2}$ atmosphere IE mRNA was induced in about $30 \%$ of the rat $9 \mathrm{G}$ cell population by addition of $50 \mu \mathrm{g} \mathrm{mL}^{-1}$ cycloheximide (Sigma, St Louis, MO, U.S.A.) for $5 \mathrm{~h}$ at $37^{\circ} \mathrm{C}$ to exponentially growing cells (Boom et al., 1986).

\section{Probes and labelling}

For the detection of $28 \mathrm{~S}$ rRNA in both HeLa and rat $9 \mathrm{G}$ cells a pGEM plasmid containing a $2 \cdot 1-\mathrm{kb}$ insert specific for the $3^{\prime}$ site of human 28S rRNA was used (Erickson et al., 1981; Bauman \& Bentvelzen, 1988). For the detection of HEF mRNA in HeLa cells, a plasmid probe containing HEF-1 complementary DNA was used (Brands et al., 1986). IE mRNA in rat $9 \mathrm{G}$ cells was detected with a genomic plasmid probe containing the 5-kb Sphl-Sall fragment of the transfected human cytomegalovirus (HCMV) IE region (Boom et al., 1986). As a negative control, HeLa and rat 9G cells were mock-hybridized with hybridization mix without probe or with nonspecific probe.

DNAs were labelled by nick-translation in the presence of digoxigenin-11-dUTP (Boehringer, Mannheim, Germany) and after $2 \mathrm{~h}$ of labelling directly precipitated with $0.3 \mathrm{M}$ sodium acetate and two volumes $100 \%$ ethanol $\left(-20^{\circ} \mathrm{C}\right)$.

\section{Fixation and pretreatment}

Cells were washed in phosphate-buffered saline pH 7.4 (PBS) and fixed at room temperature (RT) with a mixture of $4 \%(\mathrm{~V} /$ v) formaldehyde and $5 \%(\mathrm{v} / \mathrm{v})$ acetic acid in PBS for $20 \mathrm{~min}$.
After the fixation the cells were washed in PBS and treated with $0.1 \%(\mathrm{w} / \mathrm{v})$ pepsin (Sigma) in $10 \mathrm{~mm} \mathrm{HCl}$ for $1.5 \mathrm{~min}$ at $37^{\circ} \mathrm{C}$ to increase accessibility for macromolecular reagents. Cells were washed twice with double-distilled water and dehydrated in a $70-90-100 \%$ series of ethanol and stored in $70 \%$ ethanol at $4{ }^{\circ} \mathrm{C}$.

\section{In situ hybridization and immunological detection}

In situ hybridization was essentially performed as described before (Dirks et al., 1993). Digoxigenin-labelled RNA-DNA hybrids were detected with peroxidase-conjugated antidigoxigenin $\mathrm{F}(\mathrm{ab})_{2}$ fragments as described previously (Macville et al., 1995). The peroxidase reaction with $0.005 \%$ $\mathrm{H}_{2} \mathrm{O}_{2}$ and $50 \mathrm{mg} \mathrm{mL}^{-1}$ DAB (Sigma) was performed in $50 \mathrm{~mm}$ Tris $\mathrm{HCl}^{-1} \mathrm{pH} 7.4$ containing $0.01 \mathrm{~m}$ imidazole for $15 \mathrm{~min}$ in the dark.

In order to get information on the effect of scanning conditions (under fluid or dry), ethanol dehydration and air drying on the visibility of the ISH signal and the cellular structure, 28S rRNA or mock-hybridized cells were either kept in PBS after the DAB development and scanned directly in PBS (further referred to as wet cells) or dehydrated in 70 , 90 and $100 \%$ ethanol. Dehydrated cells were scanned either dry (further referred to as dry cells) or in PBS to monitor rehydration effects (further referred to as rehydrated cells). Cells hybridized for HEF or IE MRNA were directly dehydrated after the DAB development.

\section{Atomic force microscopy}

Samples were imaged with a Topometrix TMX Explorer AFM (Topometrix, Santa Clara, CA, U.S.A .) equipped with a 150$\mu \mathrm{m} \mathrm{XY}$ - and $12-\mu \mathrm{m}$ Z-scanner. The cantilever of the microscope was centred in the optical axis from a Leica DM IL (Leica, Wetzlar, Germany) inverted microscope by a home-made modification of the microscope table which allows the user to move the AFM independently of the microscope The cell of interest was positioned under the cantilever with the microscope $X Y$ translator and scanned in the contact mode with standard silicon nitride tips with a pyramidal base of $4 \mu \mathrm{m} \times 4 \mu \mathrm{m}$ and a spring constant of $0.032 \mathrm{nN}$ (Topometrix, model 1520-00). Images show either the height information or the error signal (Putman et al., 1992). The scanning conditions were similar in all the images presented here. The feedback values were optimized and not changed during the scanning.

Images were processed with the Topometrix SPMLab version 3.05 Image Analysis software (Topometrix). Processing consisted of tilt removal and adaptation of colour scalebars to mean values. Using this software, measurements on the height of the cells were taken at three different places on the cell. At least three cells per scanning condition were 
measured at equivalent points (cytoplasm, nucleolus and nucleus). For these measurements a cross-section was made through the cell including the point of interest. The data of the measurements were processed and the mean values were calculated. As the measurements were taken on a small sample the mean values indicate a tendency in the height differences of the cells rather than absolute numbers. Photographs were taken with a Polaroid HR 6000 digital palette.

\section{Results}

First the optimal scanning conditions for the detection of the specific DAB precipitate relative to cellular structures were determined. For this purpose cells, hybridized for $28 \mathrm{~S}$ rRNA or mock hybridized, were scanned wet, dry and under fluid after dehydration in ethanol (rehydrated cells). Differences between positive (Fig. 1A) and control wet cells (Fig. 1D) are the increased height of the cytoplasm, the appearance of DA B precipitate in granule-like structures (further referred to as DAB spots) in the cytoplasm and the increased height of the nucleoli of the positive cell, caused by the DAB that is precipitated at these sites. Figure $1 \mathrm{~A}$ also shows some streaking on top of the nucleus owing to material that is being removed during the scanning. Figures $1 \mathrm{C}$ and $\mathrm{IF}$ show positive and control dry cells. Nucleoli and cytoplasm of positive cells contain a large amount of DAB precipitate and are therefore much higher than in control cells. Also, the DA B spots in the cytoplasm of positive dry cells are very clear (Figs $2 \mathrm{~A}$ and $2 \mathrm{~B}$ ). When these cells are rehydrated, the difference in height between the cytoplasm of positive and control rehydrated cells is more pronounced than in wet cells. However, there is almost no height difference between the positive and control nucleoli (Figs 1B and 1E). This could not be explained by large variations in the height of nucleoli in positive cells. The nucleus, including the nucleoli, seems to be collapsed during the dehydration and is not rehydrated to its full extent (compare Figs $1 B$ and $1 A$ ). In addition, this leads to the formation of a pronounced nuclear edge. From Figs 1 and 3 it is clear that the height difference between positive and control cells is largest when the cells are dehydrated and scanned dry.

The data in Fig. 3 are the mean of a series of measurements on at least three different cells. In the case of wet and rehydrated cells a selection had to be made for relatively low and well-spread cells to avoid cantilever artefacts induced by high and steep features on the cells. Therefore, the data in Fig. 3 indicate mean differences in height between various cellular structures owing to internally precipitated DAB and/ or de- and rehydration, rather than absolute numbers.

For all scanning conditions, a remarkable difference is observed between rat $9 \mathrm{G}$ cells and HeLa cells concerning the height of the nucleoli in positive and control cells. While there exists a remarkable height difference between the nucleoli of positive and control rat $9 \mathrm{G}$ cells, this is almost absent in HeLa cells (Fig.3), although light microscopical observation of positive HeLa cells revealed DA B precipitate in the nucleoli (data not shown). Nevertheless, a prominent difference in the height and the structure of the cytoplasm is found between positive and control HeLa cells. The influence of dehydration and rehydration on HeLa cells is similar to the effects on rat $9 \mathrm{G}$ cells (Figs $3,4 \mathrm{~A}$ and $4 \mathrm{~B}$ ).

Because it is clear from these experiments that dry scanning provides the most sensitive detection of internally precipitated DAB, rat $9 \mathrm{G}$ cells hybridized for IE MRNA and HeLa cells hybridized for HEF MRNA cells were dehydrated directly after DAB development and scanned dry. Rat $9 \mathrm{G}$ cells hybridized for IE mRNA revealed clear cytoplasmic signals in $\approx 20-30 \%$ of the cells (Fig. 5). The cytoplasm of positive cells contained DAB spots, in contrast to the smooth cytoplasm of the negative cells. Because the cells were heat-denatured before the ISH procedure, a nuclear signal from the integrated viral DNA is visible in all cells. Owing to the accumulation of RNA at or near the site of transcription, the nuclear signal in the positive cells is much larger than the nuclear signal in the negative cells (Fig. 5; see also Dirks et al., 1993).

The signal of the least abundant mRNA detected in this study, HEF, is clearly visible in dry cells. The signal appears as DAB spots in the cytoplasm (Figs6A and 6B) similar to the appearance of the signal in cells hybridized for $28 \mathrm{~S}$ rRNA, although the cytoplasm of HEF hybridized cells is much lower. No nuclear signal from the HEF gene and HEF transcripts can be seen because HEF transcription at steady state is most probably too low to be detected by AFM.

\section{Discussion}

In order to investigate the value of AFM for the visualization of internal cellular molecules, we used an RNA in situ hybridization (RNA - ISH) procedure to gen erate cytoplasmic, nucleolar and nuclear DAB signals. To determine the influence of the size of the generated DAB signal on the detection efficiency, RNAs with different expression levels were used. 28S rRNA is located inside the nucleolus and cytoplasm of cells and is expressed abundantly in both rat $9 \mathrm{G}$

Fig. 1. Height images of rat $9 \mathrm{G}$ cells. Rat $9 \mathrm{G}$ cells were hybridized for $28 \mathrm{~S}$ rRNA or mock-hybridized. Haptenized hybrids were detected with a peroxidase-labelled antibody and subsequent development with DA B. A-C, cells after hybridization for 28S rNA; D-E, mock hybridized cells. A and $\mathrm{D}$, wet rat $9 \mathrm{G}$ cells, colour scale bar $=2999 \mathrm{~nm} ; \mathrm{B}$ and $\mathrm{E}$, rehydrated rat $9 \mathrm{G}$ cells, colour scale bar $=1999 \mathrm{~nm} ; \mathrm{C}$ and F, dry rat $9 \mathrm{G}$ cells, scale bar $=999 \mathrm{~nm}$. Scale bars A, B, D-F, $5 \mu \mathrm{m} ; \mathrm{C}, 3 \mu \mathrm{m}$. 

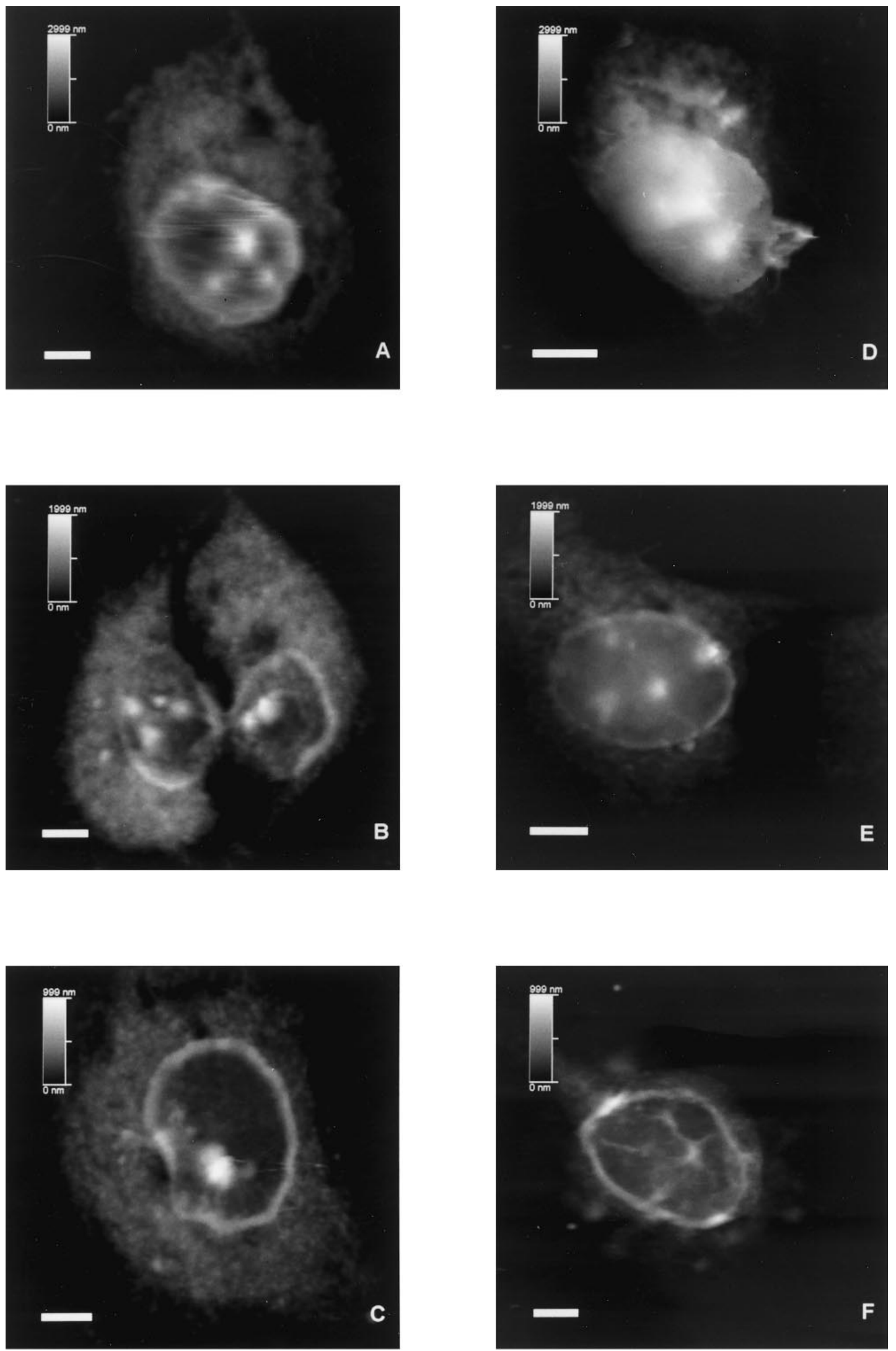

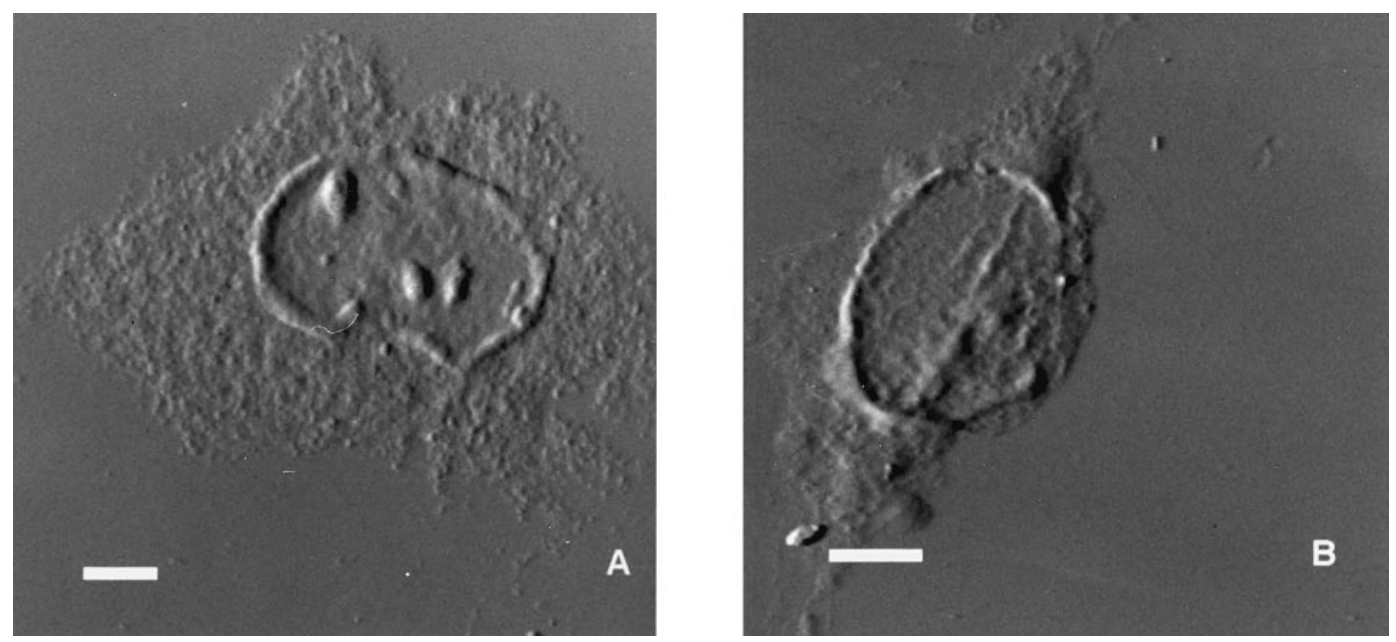

Fig. 2. Error-signal image of dry rat $9 \mathrm{G}$ cells hybridized for $28 \mathrm{~S}$ rRNA or mock-hybridized. The deflection of the cantilever is represented in errorsignal images. White represents upward slopes, black represents downward slopes. A, 28S rRNA hybridized cell; B, mock hybridized cell. Note the DAB spots in the cytoplasm of the cell in A. Scale bars, $5 \mu \mathrm{m}$.

and HeLa cells. Furthermore, rat $9 \mathrm{G}$ cells carry, stably integrated in the host genome, a tandem repeat of $\approx 10$ copies of plasmid pES containing HCMV IEA DNA. IE transcription is repressed normally but it can be induced by cycloheximide treatment. After release from the cycloheximide block, $\approx 30 \%$ of the nuclei show expression of the $72 \mathrm{~K} \mathrm{IE}$ antigen as detected with immunofluorescence(Boom et al., 1986, 1988; Geelen et al., 1987). HEF mRNA is the least abundant of the RNAs investigated in this study and localizes solely in the cytoplasm of HeLa cells. In this study we tested whether the high resolution of AFM could give more information about the localization of such RNA molecules compared with light microscopy.

Nucleolar and cytoplasmic signals from the 28S rRNA hybridization are not very pronounced in wet cells. Some nucleolus-like structures are visible on the nucleus of positive wet cells. Because wet cells are relatively soft and the force exerted locally by the tip can be very high owing to the height and steepness of the sample, material is being removed by the tip. This results in streaking of the image in the scan direction. Measurements and calculations on the deformation of various materials indicate that imaging forces of about $10^{-11} \mathrm{~N}$ should be used for nondestructive imaging of biological samples with nanometre resolution (Weisenhorn et al., 1993). This implies an imaging force two orders of magnitude lower than routinely used whileimaging under liquid.

Because of the softness of the sample it is conceivable that deformation of the cellular and nuclear membrane occurs owing to the scanning force. The deformation would be much less in areas where an internal DAB precipitate is present or where internal structures with a relatively rigid structure are lying. Therefore, DAB signals in nucleoli and the cytoplasm of wet cells are still visible, albeit much less than in rehydrated or dry cells. Although the nucleoli of HeLa cells did contain DAB precipitate, the difference in nucleoli heights between positive and control cells is not clear. This is most probably caused by either the volume or the structure of the nucleoli in these cells. Because control wet cells also show high nucleoli it is conceivable that the nucleoli of HeLa cells are either very rigid or very large. Therefore, the effect of the scanning force or internally precipitated DA B on the visualization of these nucleoli would be much less. It is known that variations in the volume and arrangement of nucleolar components between different cell types occur (reviewed by Wachtler \& Stahl, 1993).

When the cells were dehydrated in an ethanol series and rehydrated with PBS and then scanned in PBS, some interesting details appeared. Because of the dehydration the cell height decreased significantly and never fully recovered during the rehydration in PBS. The main difference between wet and rehydrated cells is the better visibility of the internal cytoplasmic ISH signal in rehydrated cells. The absence of a clear difference in nucleoli heights between rehydrated positive and control cells could be induced by either a dehydration effect or variations in rehydration between cellular compartments due to structural differences or the presence of DAB precipitate. Furthermore, the appearance of a nuclear edge can be seen, probably caused by differences in rehydration between the DAB-filled cytoplasm and the nucleus. The rehydrated cells resemble the dry cells but are much higher. Scanning rehydrated cells results in improved images because of reduced height and extra fixation during dehydration and air-drying of the cells. However, the cells are by no means fully rehydrated and do not resemble wet cells. Therefore, extreme caution should be taken when drawing conclusions about the in vivo situation with data obtained from rehydrated cells. 


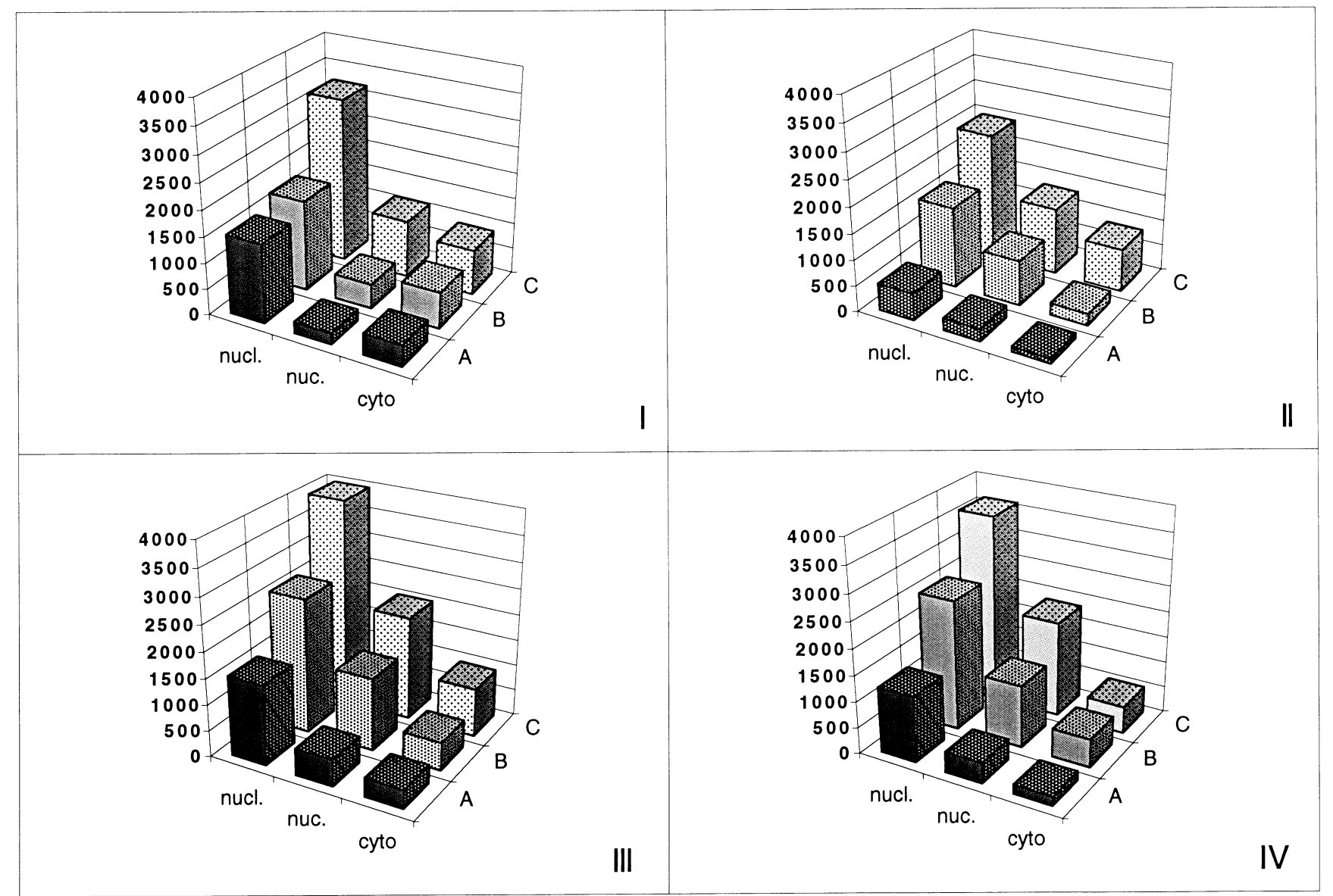

Fig. 3. Height measurements on wet, rehydrated and dry rat $9 \mathrm{G}$ and HeLa cells after either hybridization for $28 \mathrm{~S}$ rRNA or mock-hybridization. Measurements were taken at three places (nucleus, nucleolus and cytoplasm) on at least three different cells. Heights of the bars in the histogram are the mean of these measurements and represent a tendency in the height of the different cellular components rather than actual numbers. I, rat $9 \mathrm{G}$ cells after $28 \mathrm{~S}$ rRNA hybridization; II, mock-hybridized rat $9 \mathrm{G}$ cells; III, HeLa cells after $28 \mathrm{~S}$ rRNA hybridization; IV, mock-hybridized HeLa cells. Nucl = nucleoli; nuc = nucleus; cyto=cytoplasm. A, dry cells; B, rehydrated cells; C, wet cells.
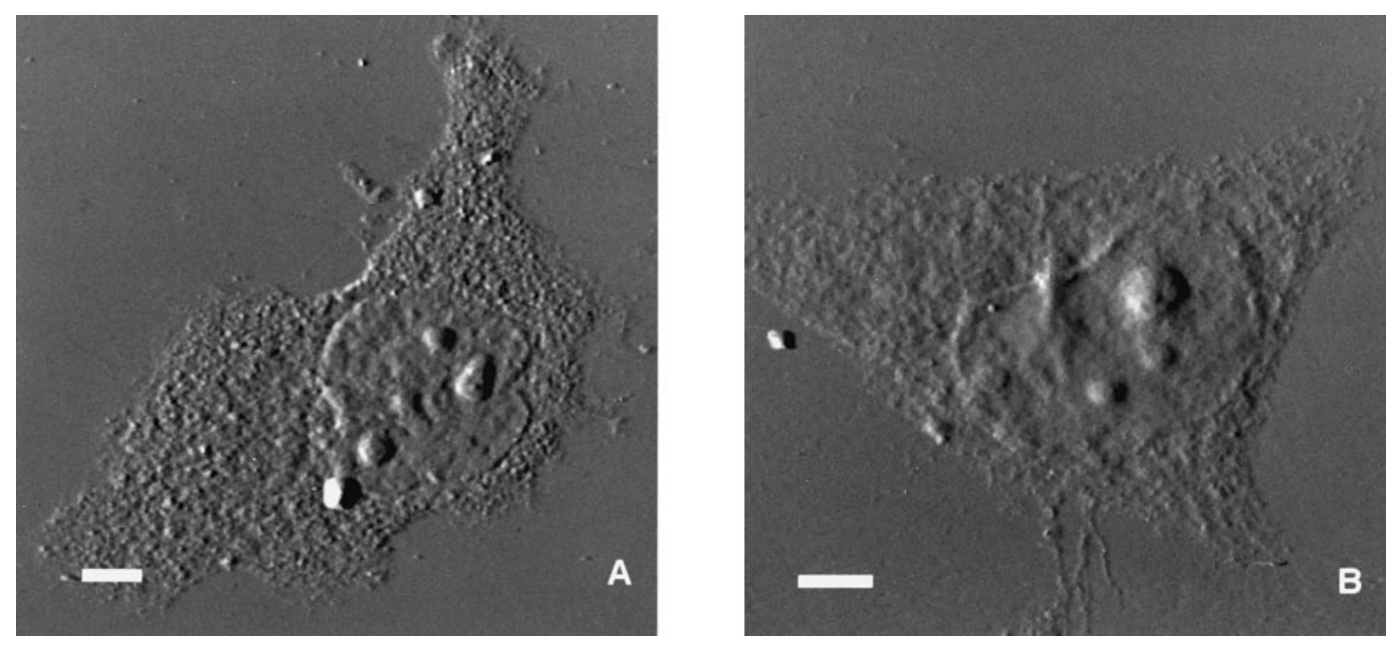

Fig. 4. Error-signal image of dry HeLa cells hybridized for $28 \mathrm{~S}$ rRNA or mock-hybridized. A, $28 \mathrm{~S}$ rRNA hybridized cell; B, mock hybridized cell. Note the DAB spots in the cytoplasm of the cell in A. Scale bars, $5 \mu \mathrm{m}$. 


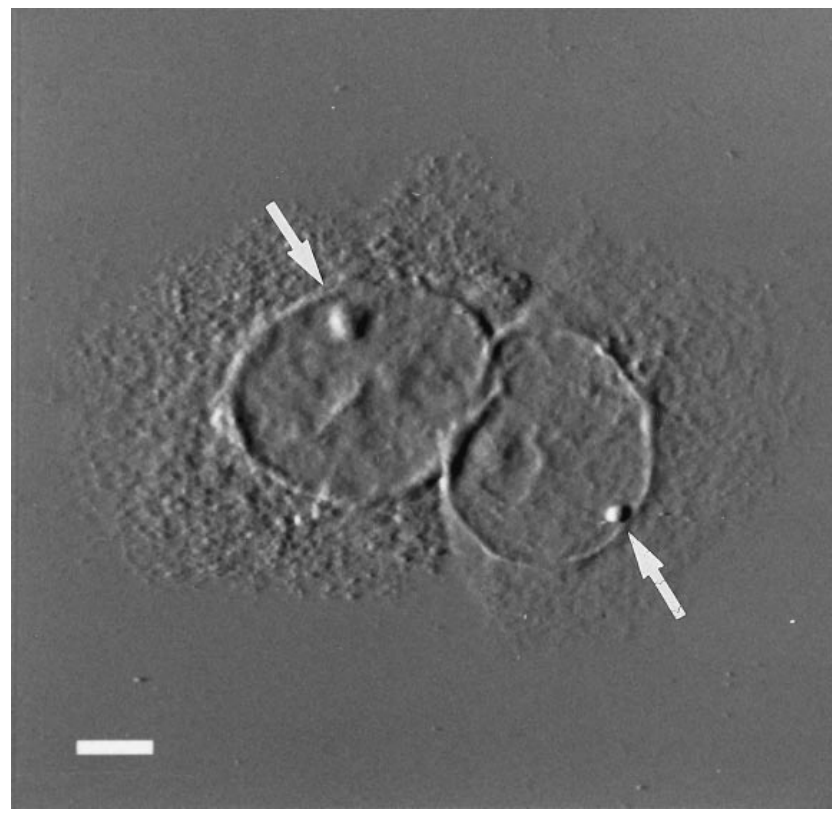

Fig. 5. Error-signal image of two rat $9 \mathrm{G}$ cells hybridized for IE mRNA. The cell on the left clearly shows DAB spots in the cytoplasm. Arrows indicate the nuclear signals. Note the larger size of the nuclear signal in the cell on the left because of accumulated RNAs at or near the site of transcription. Scale bars $=5 \mu \mathrm{m}$.

When cells are scanned dry, the hybridization signals of the 28S rRNA, the IE and HEF MRNA can easily be seen (Figs 5 and 6). Unfortunately, small nuclear signals from the IE mRNA in transport from the transcription site to the nuclear envelope cannot be seen (Macville et al., 1995). These signals are probably too small to be detected with AFM. The negative cell in Fig. 5 shows the same features as the cell in Fig. 1F. Only the nuclear DNA signal can be seen in this cell. The nuclear signal in HeLa cells hybridized with the
HEF probe is also too small for detection with AFM. When the sensitivity of AFM is compared with conventional light microscopical observations it is clear that the sensitivity is similar for the RNAs used in this study. However, the sensitivity of AFM is lowered by the difficulties in distinguishing background spots from the specific spots and by the fact that the DAB spot has to have a certain size to be visible from the outside of the cell. It is clear from these results that the most sensitive detection of internal DAB precipitate with AFM will occur when cells are dehydrated and scanned dry.

Our findings indicate that internally generated DAB signals can be seen with AFM provided they are large enough to exceed the biological background. Because of this, an optimal balance has to be found between the size of the generated signal and the highest resolution. Furthermore, when the signals are relatively small, it is essential to know the localization of the signals beforehand. Alternatively, a newly developed cytochemical enhancement system to increase the signal could be used, but will most probably decrease the resolution of AFM (Raap et al., 1995). This is a limitation for the use of AFM for this type of study. Although there is a small increase in spatial resolution between AFM and optical or confocal microscopy, this difference is much smaller than expected on the basis of the physical differences in imaging technique However, AFM may be very useful when small mRNA signals have to be detected in cellular protrusions like microvilli or pseudopodia. It has been shown that cellular protrusions, when they do not contain labelled cellular components, are difficult to detect by fluorescence microscopy, while they can easily be imaged by AFM (Putman et al., 1993c). In the case of mRNAs which localize specifically in these protrusions, like mRNAs for cytoskeleton proteins (Lawrence \& Singer, 1986), the AFM can be used to image both the mRNA after RNA-ISH
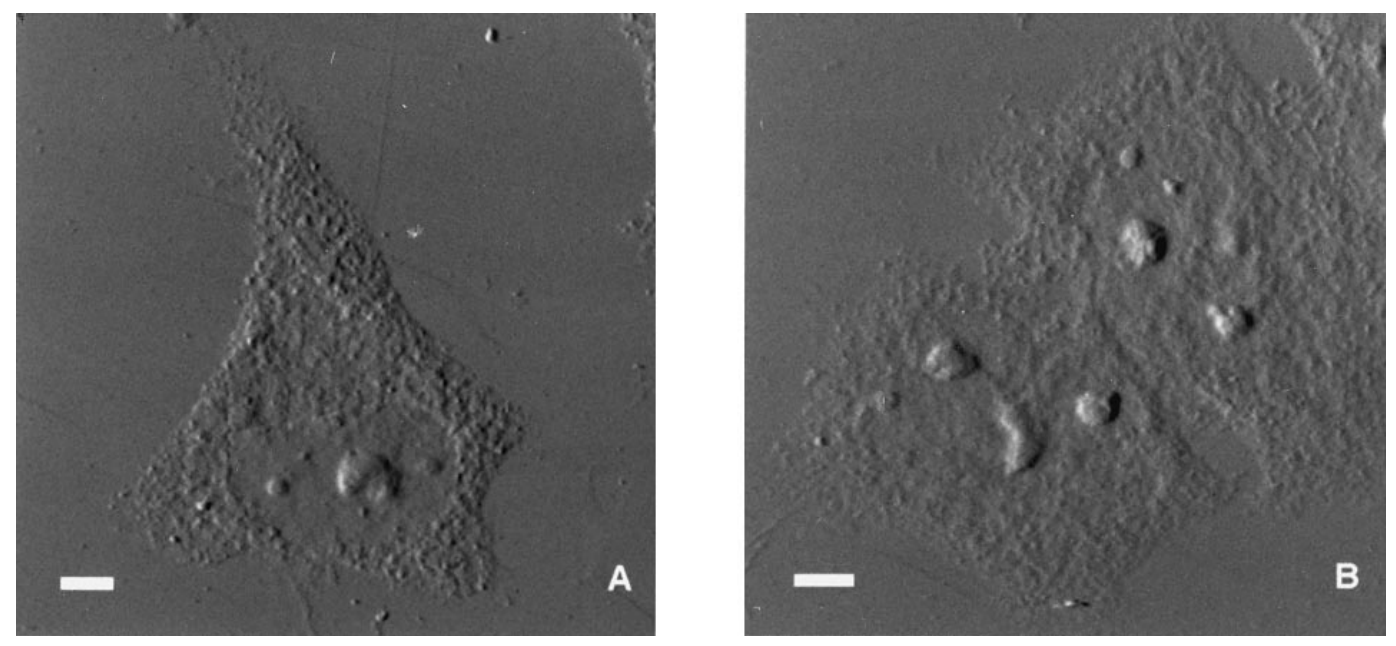

Fig. 6. Error-signal image of HeLa cells hybridized for HEF mRNA or mock-hybridized. A, HEF hybridized HeLa cell; B, mock-hybridized HeLa cell. Cytoplasmic DAB spots can easily be seen in A. Scale bars $=5 \mu \mathrm{m}$. 
and cytochemical detection and the cellular substructure where the signal is localized.

\section{Acknowledgments}

We thank Dr R. W. Dirks for critically reading the manuscript and meaningful discussion. This research was financially supported by the Dutch Organization for Scientific Research (NWO), grant numbers 900-534-107, 900-538-041, 900534-079 and 900-534-109.

\section{References}

Bauman, J.G.J. \& Bentvelzen, P. (1988) Flow cytometric detection of ribosomal RNA in suspended cells by fluorescent in situ hybridization. Cytometry, 9, 517-524.

Binnig, G., Quate, C.F. \& Gerber, C. (1986) A tomic force microscope. Phys. Rev. Lett. 3, 1281-1286.

Boom, R., Geelen, J.L., Sol, C.J., Raap, A.K., Minnaar, R.P., Klaver, B.P. \& van der Noorda (1986) Establishment of a rat cell line inducible for the expression of human cytomegalovirus immediate-early gene products by protein synthesis inhibition. J. Virol. 58, 851-859.

Boom, R., Sol, C.J., Minnaar, R.P., Geelen, J.L., Raap, A.K. \& van der Noorda (1988) Induction of gene expression under human cytomegalovirus immediate early enhancer control by inhibition of protein synthesis is cell cycle dependent. J. Gen. Virol. 69, 1179-1193.

Brands, J.H.G.M., Maassen, J.A., van Hemert, F.J., Amons, R. \& Möller, W. (1986) The primary structure of the I subunit of human elongation factor 1 . Structural aspects of guaninenucleotide-binding sites. Eur. J. Biochem. 155, 167-171.

Dirks, R.W., van de Rijke, F.M., Fujishita, S., van der Ploeg, M. \& Raap, A.K. (1993) Methodologies for specific intron and exon localization in cultured cells by haptenized and fluorochromized probes. J. Cell Sci. 104, 1187-1197.

Erickson, J.M., Rushford, C.L., Dorney, D.J. \& Wilson, G.N. (1981) Structure and variation of human ribosomal DNA: molecular analysis of cloned fragments. Gene, 16, 1-19.

Geelen, J.L.M.C., Boom, R., Klaver, G.P.M., Minnaar, R.P., Feltkamp, M.C.W., van Milligen, F.J., Sol, C.J.A \& van der Noorda (1987) Transcriptional activation of the major immediate early transcription unit of human cytomegalovirus by heat-shock, arsenite and protein synthesis inhibitors. J. Gen. Virol. 68, 2925-2931.

Hansma, H.G. \& Hoh, J.H. (1994) Biomolecular imaging with the atomic force microscope. Ann. Rev. Biophys. Biomol. Struct. 23, 115-139.
Henderson, E., Haydon, P.G. \& Sakaguchi, D.S. (1992) Actin filament dynamics in living glial cells imaged by atomic force microscopy. Science, 257, 1944- 1946.

Keller, D., Chang, L., Luo, K., Singh, S. \& Yorgancioglu, M. (1992) Scanning force microscopy of cells and membrane proteins. Proc. SPIE, 1639, 91-101.

Lawrence, J.B \& Singer, R.H. (1986) Intracellular localization of messenger RNAs for cytoskeletal proteins. Cell, 4, 407415.

Macville, M.V.E, Wiesmeijer, K.C., Dirks, R.W., Fransen, J.A.M. \& Raap, A.K. (1995) Saponin pre-treatment in pre-embedding electron microscopic in situ hybridization for detection of specific RNA sequences in cultured cells: a methodological study. J. Histochem. Cytochem. 40, 1005-1018.

Pietrasanta, L.I., Schaper, A. \& Jovin, T.M. (1994) Imaging subcellular structures of rat mammary carcinoma cells by scanning force microscopy. J. Cell Sci. 107, 2427-2437.

Putman, C.A.J., de Grooth, B.G., Hansma, P.K. \& van Hulst, N.F. (1993a) Immunogold labels: cell surface markers in atomic force microscopy. UItramicroscopy, 48, 177-182.

Putman, C.A.J., de Grooth, B.G., Wiegant, J.C.A.G. \& Raap, A.K. (1993b) Detection of in situ hybridization to human chromosomes with the atomic force microscope. Cytometry, 14, 356361.

Putman, C.A.J., van Leeuwen, A.M., de Grooth, B.G., Radosevic, van de Werf, C.O., van Hulst, N.F \& Greve, J. (1993c) A tomic force microscopy combined with confocal laser scanning microscopy: a new look at cells. Bioimaging, 1, 63-70.

Putman, C.A.J., van de Werf, K.O., de Grooth, B.G., van Hulst, N.F., Greve, J. \& Hansma, P.K. (1992) A new imaging mode in atomic force microscopy based on the error signal. Proc. SPIE, 1936, 198-204.

Raap, A.K., van de Corput, M.P.C., Vervenne, R.A.W., van Gijlswijk, R.P.M., Tanke, H.J. \& Wiegant, J. (1995) Ultra-sensitive FISH using peroxidase mediated deposition of biotin- or fluorochrome tyramides. Hum. Mol. Genet. 4, 529-534.

Rasch, P., Wiedemann, U., Wienberg, J. \& Heckl, W.M. (1993) Analysis of banded human chromosomes, in situ hybridization patterns by scanning force microscopy. Proc. Natl. Acad. Sci. USA, 90, 2509-2511.

Wachtler, F. \& Stahl, A. (1993) The nucleolus: a structural and functional interpretation. M icron, 24, 473-505.

Weisenhorn, A.L., Khorsandi, M., Kasas, S., Gotzos, V. \& Butt, H-J. (1993) Deformation and height anomaly of soft surfaces studied with an AFM. Nanotechnology, 4, 106-113.

Yang, J. \& Shao, Z.F. (1995) Recent advances in biological atomic force microscopy. Micron, 26, 35-49. 